\title{
Suppression of scroll wave turbulence by noise
}

\author{
S. Alonso, ${ }^{1, *}$ J. M. Sancho, ${ }^{2}$ and F. Sagués ${ }^{1}$ \\ ${ }^{1}$ Departament de Química Física, Universitat de Barcelona, Avenida Diagonal 647, 08028 Barcelona, Spain \\ ${ }^{2}$ Departament d'Estructura $i$ Constituents de la Matèria, Universitat de Barcelona, Diagonal 647, E-08028 Barcelona, Spain
}

(Received 13 February 2004; revised manuscript received 3 May 2004; published 2 December 2004)

Rotating scroll waves are dynamical spatiotemporal structures characteristic of three-dimensional active media. It is well known that, under low excitability conditions, scroll waves develop an intrinsically unstable dynamical regime that leads to a highly disorganized pattern of wave propagation. Such a "turbulent" state bears some resemblance to fibrillation states in cardiac tissue. We show here that this unstable regime can be controlled by using a spatially distributed random forcing superimposed on a control parameter of the system. Our results are obtained from numerical simulations but an explicit analytical argument that rationalizes our observations is also presented.

DOI: 10.1103/PhysRevE.70.067201

PACS number(s): 82.40.Bj, 05.40.Ca, 47.54.+r

Excitable media may propagate a rich variety of wave structures, a scroll wave being a particular form characteristic of three-dimensional systems [1]. This structure has special interest in the dynamics of the cardiac tissue. Actually, scroll waves have often been related to cardiac arrhythmias and their eventual degeneration into a turbulent regime has been sometimes invoked as a possible mechanism to understand the fibrillating state of the heart, preceding cardiac death [2]. Therefore, studies of how to control this instability phenomenon are worth performing.

In its traverse cross section a scroll wave looks like a spiral. Such spirals are stacked one upon another, with the free edge of the scroll wave rotating around an unexcitable filament. This filament may be straight or curved; it may also form closed rings that, depending on the conditions of the medium, may either contract or expand, or even form more complicated entangled loops [3]. The stability of this filament will principally determine the dynamic regimes of the whole wave structure. In particular, under low excitability conditions, the straight filament is known to be unstable, i.e., it is endowed with a negative tension (see below), leading to a highly disorganized wave propagation regime. Actually, such a scenario constitutes a particular route to what we have recently referred to as Winfree turbulence [4,5]. Our aim here is to study the stability of scroll waves when they propagate under external fluctuations of the medium excitability. We will eventually demonstrate, rather counterintuitively, that episodes of chaotic wave propagation can indeed be suppressed by using a spatially distributed random external forcing.

Previous numerical research dealing with nonresonant periodic global modulation of such turbulent states, demonstrated that it is possible to suppress these chaotic episodes, resetting the forced weak excitable system into its normal conducting conditions [4]. Here, although the outcome will be similar, the mechanism is completely different, and this is a key point of this Brief Report. In the case of time periodic uniform forcing it amounts to a near resonant coupling be-

\footnotetext{
${ }^{*}$ Corresponding author. Email address: s.alonso@qf.ub.es
}

tween the forcing modulation and the intrinsic rotation mode of the scroll wave [4]. Here, however, the rationale to interpret our results will be a change in the filament tension as a genuine effect of the external spatiotemporal noise.

Examples of excitation waves are ubiquitous in nature or in laboratory-tailored experiments. Waves of chemical concentrations for bulk autocatalytic or surface reactions [6], pulses of the so-called action potential in the previously referred cardiological context [7], or accumulated passing profiles of signaling agents, triggering aggregation in populations of Dyctiostelium discoideum [8], are among the most well-documented realizations. To encompass as many as possible of the above-mentioned scenarios and to prove the generic nature of the proposed mechanism of taming scroll wave turbulence by noise, the results here reported refer to the simplest scheme of an excitable system, as represented by the so-called Barkley model [9].

As a direct antecedent of our study here, experimental and numerical studies abound reporting different manifestations of what could be opportunely qualified as an ordering role of external noise when acting on extended dynamic systems [10]. There is no mystery in these counterintuitive phenomena, but rather these findings reveal disparate signatures of the delicate coupling between noise and the nonlinearities of most of these systems. We leave apart the context of summed up additive noise-triggered resonant or coherent effects in connected lattices, to concentrate on more genuine features of parametric (multiplicative) noise acting on patternsupporting systems. Focusing purely on wave phenomena, it has been proved analytically and found numerically and experimentally, mainly using the photosensitive BelousovZhabotinsky reaction [11-13], that external fluctuations may allow the propagation of one-dimensional (1D) waves in nonexcitable media [13], sustain the propagation of pieces of waves, favor the growth of spiral waves in subexcitable media [11,13-17], and even generate target patterns, mimicking oscillatory conditions, in strictly excitable media [13]. Several of these effects can be interpreted by assuming an increase of the effective excitability of the media due to the spatiotemporal stochastic forcing. However, it is fair to say that this conclusion may largely depend on the specific way the external noise enters into the intrinsic considered dynam- 
ics. As a matter of fact the reverse situation of a decreased excitability has been also very recently demonstrated [18].

All these studies, however, refer to two-dimensional systems, the only exception, to our knowledge, being a few preliminary reports of noise effects on three-dimensional systems $[19,20]$, devoted, respectively to the role of noise either in nucleating waves or in extending the lifetime of scroll rings.

As a minimum scheme for an excitable system, the equations of the Barkley model [9] under parametric noise forcing read

$$
\begin{gathered}
\partial_{t} u=D_{u} \nabla^{2} u+\frac{1}{\epsilon} u(1-u)\left(u-\frac{v+b+\xi(\mathbf{x}, t)}{a}\right), \\
\partial_{t} v=u-v .
\end{gathered}
$$

We have chosen to introduce the noise through the parameter $b \rightarrow b+\xi(\mathbf{x}, t)$, since this parameter is singularly relevant in determining the excitability of the medium. The stochastic process $\xi(\mathbf{x}, t)$ is assumed to be a Gaussian white noise with zero mean and correlation,

$$
\left\langle\xi(\mathbf{x}, t) \xi\left(\mathbf{x}^{\prime}, t^{\prime}\right)\right\rangle=2 \sigma^{2} \delta\left(t-t^{\prime}\right) \delta\left(\mathbf{x}-\mathbf{x}^{\prime}\right) .
$$

Notice that the noise is spatiotemporally distributed, uncorrelated, and coupled to the dynamics of the system nonlinearly. For the numerical simulation of these equations we have discretized Eq. (1) in a 3D grid and numerically integrated the corresponding set of ordinary stochastic differential equations, using finite spatial differences with a 19-point prescription for the Laplacian, and the explicit first order Euler method for the time variable [10]. No flux boundaries conditions were used throughout the whole numerical study.

Our objective is to study this model under conditions of weak excitability, actually near the subexcitable limit beyond which waves are no longer supported by the media and decay. It is worth emphasizing at this point that under these conditions, spiral waves in two dimensions are completely stable but filaments of the 3D scroll waves are unstable. This means that a nonperfectly straight filament, no matter how much distorted it may be, starts to snake and bend around, giving rise to an endlessly growing entangled coil. Nevertheless, the filament remains intact until it reaches the boundaries, where it breaks [5,21]. This regime is represented in the first four spots of Fig. 1 for an initially straight untwisted filament with the two ends located at the upper and lower plane boundaries of a cubic slab. For larger times, the system would display a complicated and incoherent pattern of wave propagation which evokes the fibrillation regime of a cardiac tissue.

Indeed, in the same Fig. 1 we can see the effects of the stochastic forcing (the last six spots). It appears clearly that after the introduction of the external noise the instability is eliminated and the remaining pieces of the filament stretch out to adopt straight conformations with small random motions. This effect will be possible, however, only for a limited range of noise intensities. For high enough intensities the noise would perturb the full 3D system and waves would

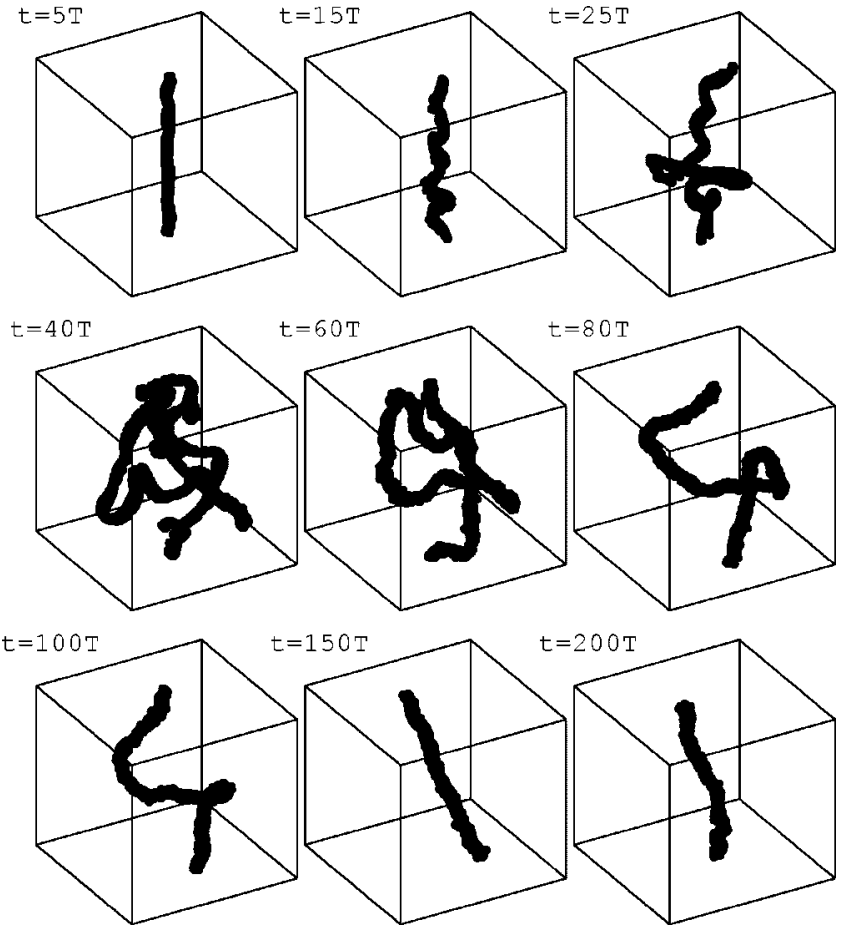

FIG. 1. Evolution of an initially unstable straight filament. At time $t=40 T$, a random forcing is added to the medium. Further evolution of the system goes in the direction of filament stabilization. Points pertaining to the filament are calculated using the prescription $u_{f}=1 / 2$ and $\partial_{t} u_{f}=0$ during a whole period of rotation of the scroll wave around the vortex filament $(T=4.5)$. The corresponding observation time is shown in each snapshot. Model parameter values are $a=1.1, b=0.16, \epsilon=0.02$, and $D=1$. Simulation was run in a box of $150 \times 150 \times 150$ pixels with $\Delta x=0.4$ and $\Delta t$ $=0.01$, using nondimensional distance and time units.

be randomly generated through all the system, giving rise to a noisy disorganized pattern of wave propagation.

In Fig. 2 it is shown that such a stabilization mechanism is generic and independent of the particular topology of the filament. There, we reproduce several snapshots corresponding to the dynamical evolution of a scroll ring. For zero or small noise intensity the scroll ring develops the turbulent regime and breaks out when it hits the boundaries. On increasing the intensity of the fluctuations, the scroll ring is marginally stabilized or more generally forced to eventually shrink and disappear.

Let us introduce now the theoretical framework to analyze these results. To describe the dynamics of the filament one may endow it with a linear tension $\alpha$ in such a way that, as a first approximation, the radius of any of its curved portions evolves with a rate proportional to its curvature [22],

$$
\frac{d R}{d t}=-\frac{\alpha}{R} .
$$

This tension is usually considered positive, but it has been found that there is a wide region in the parameter space of generic excitable media, corresponding precisely to weak excitability, where it is negative $[5,21]$. 


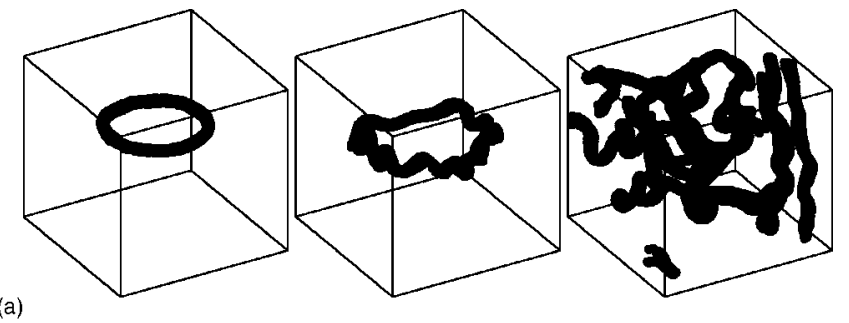

(a)

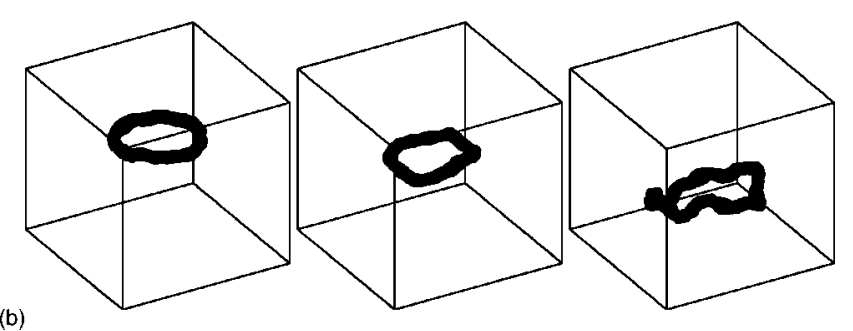

(b)

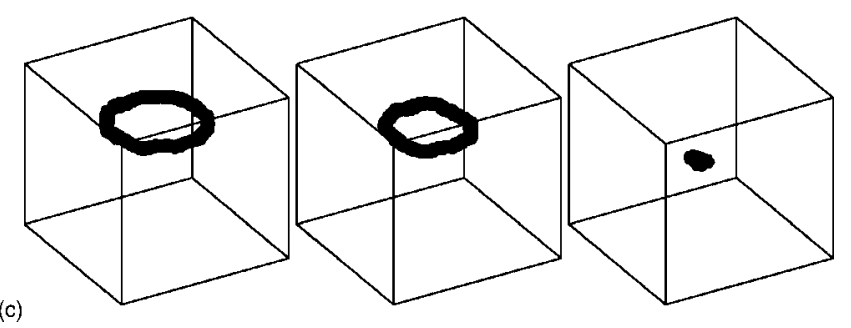

FIG. 2. An initially ringlike filament (expanding in absence of noise) evolving for three different noise intensities: $\sigma^{2}=0.00002$ (a), 0.0005 (b), and 0.0013 (c). Snapshots correspond to 5, 20, and 60 periods of rotation. Model parameters as in Fig. 1. Simulations performed in a box of $210 \times 210 \times 210$ with $\Delta x=0.3$ and $\Delta t=0.01$.

A quantitative description of the evolution of the filament under random forcing is presented in Fig. 3. There, and taking the averaged radius as indicator, we show a situation of an initially expanding scroll ring, later on prevented to destabilize under the externally imposed random forcing. Within our theoretical scheme, the whole dynamics of the

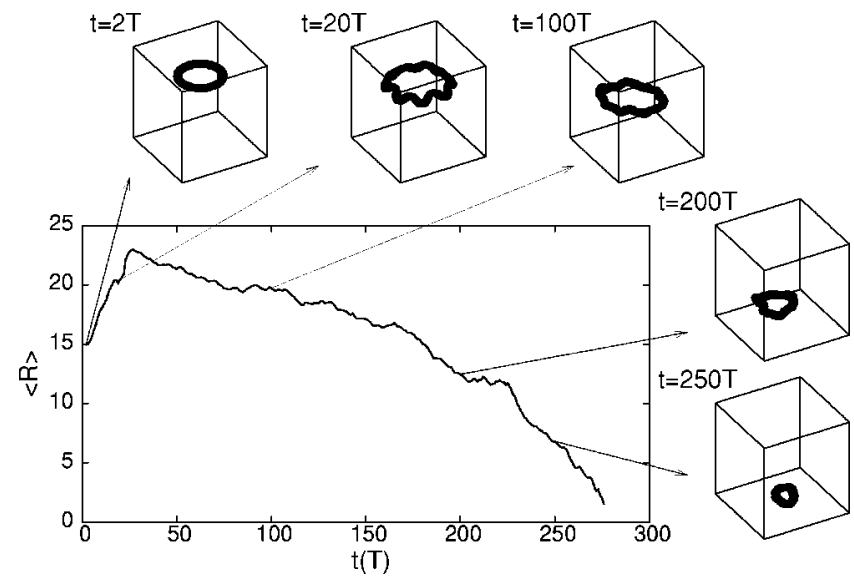

FIG. 3. Evolution of the mean radius of an initially unstable scroll ring. At $t=22 T$ a white noise $\left(\sigma^{2}=0.0002\right)$ is imposed on the system. Panels of the snapshots of the filament are also shown. Model parameters as in Fig. 1. Simulation conducted in a box of $160 \times 160 \times 250$ with $\Delta x=0.4$ and $\Delta t=0.01$.

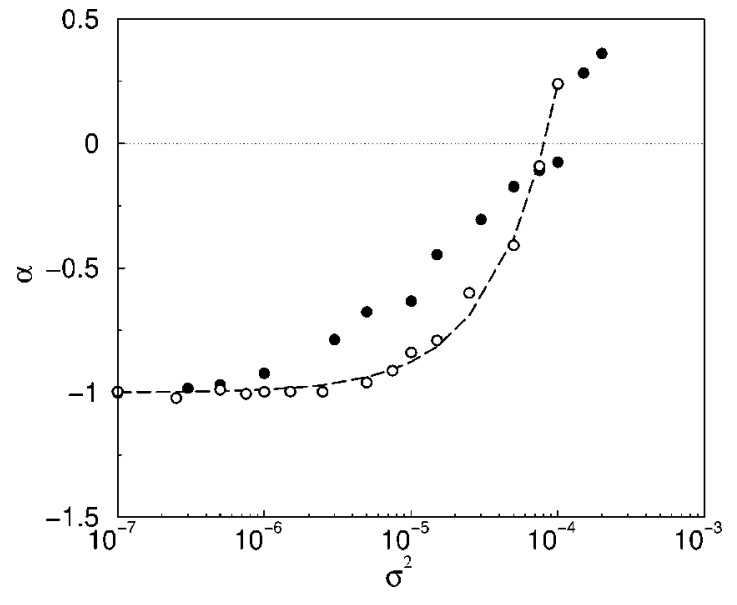

FIG. 4. The filament tension $\alpha$ as a function of the noise intensity. Values are estimated by fitting the numerical simulation results with the analytical solution of Eq. (3), $R^{2} \sim 2 \alpha t$, during the first 15 periods of rotation. Full circles $(0)$ stand for values obtained by averaging over ten realizations of the randomly forced activatorinhibitor system as given by Eq. (1). Open circles $(O)$ correspond to values obtained from simulations of the deterministic effective model with parameters renormalized according to Eq. (5). Dashed line corresponds to a linear fit (slope 12 500) of the effective filament tension. Model parameters as in Fig. 1. Simulation conducted in a box of $160 \times 160 \times 160$ with $\Delta x=0.4$ and $\Delta t=0.01$.

filament of a scroll wave is governed by the tension parameter defined in Eq. (3). Thus it is natural to expect a profound change in the parameter $\alpha$ under the effect of noise and in this way we hope to rationalize the results above. One can even predict that such a parameter may change its sign, turning an expanding, and eventually turbulent dynamics of a scroll wave into a stretching one. In fact a set of systematic simulations, conducted for an expanding scroll ring when varying the intensity of the fluctuations, enables us to construct Fig. 4, reproducing the results of $\alpha$ versus $\sigma^{2}$. Notice how $\alpha$ does change sign for an intermediate value of the noise intensity. Actually we had somewhat anticipated this result in the sense that the main influence of the noise in our system is to enhance the excitability of the medium.

Let see now how this interpretation is related to the original stochastic model. The noise effect can be explained theoretically by analyzing the statistical properties of the random term in the equations of motion (1). Since we are assuming that the noise has an external origin, thus necessarily with finite time and length correlations but approximated here as a white noise, we are led to interpret these stochastic deferential equations in the Stratonovich sense. Within this latter formulation, an standard calculation, restricted to the lowest order in the noise intensity, enables us to extract a nonzero term from the averaged equation for the activator concentration. Such a systematic contribution is implicitly evaluated as

$$
\langle u(1-u) \xi(\mathbf{x}, t)\rangle=\frac{\sigma^{2}}{\Delta x^{3}}\langle u(1-u)(1-2 u)\rangle .
$$

The remaining task is to obtain an effective equation that can absorb such a term while retaining a new noise term with 
zero mean value [10]. In particular, given the polynomial form of the reactive terms of the Barkley model, this procedure is straightforward and amounts simply to a renormalization of the parameters of the system [16]:

$$
\begin{gathered}
a^{\prime}=a\left(1-\frac{2 C(0)}{\epsilon a^{2}}\right), \quad b^{\prime}=b-\frac{C(0)}{\epsilon a}, \\
\epsilon^{\prime}=\frac{\epsilon}{\left(1-2 C(0) / \epsilon a^{2}\right)},
\end{gathered}
$$

where $C(0)=\sigma^{2} / \Delta x^{3}$ is the local noise intensity in each lattice cell. This property of the model makes easier the analysis and prediction of the noise effects. In fact, the noise now modifies the medium which, with the new parameters, is effectively located into zones of higher excitability of the parameter space. It is worth remarking here that, with $a$ of order 1 but $b$ one order of magnitude smaller, the noise correction to the parameter $b$, controlling the medium excitability, is the largest one in Eq. (5).

This analysis is supported also by the following complementary numerical test. For some values of the noise intensity we evaluate the effective parameters using Eq. (5). With these new parameters we simulate Eqs. (1) for scroll rings without noise, and evaluate the corresponding parameter $\alpha$. The results obtained are also reproduced in Fig. 4. It is clear that the stochastic model (1) behaves qualitatively as predicted by the deterministic renormalized model, confirming our interpretation of the way the noise affects the system. Quantitative differences are attributed to an increase of the filament roughness which renders the practical calculation of $\alpha$ more difficult (see caption of Fig. 4).

Summarizing the results of this Brief Report, we have shown that the instability of scroll waves which appears in three-dimensional excitable systems in the low excitability regime can be tamed by feeding spatiotemporal stochastic fluctuations into one of the parameters which controls the excitability of the system. This is an alternative suppression procedure to the nonresonant periodic forcing method introduced in Ref. [4]. Although our results are mostly numerical, a theoretical analysis has been presented to reveal the origin of such a random forcing-based control. This method could have potential interest in studies of cardiac fibrillation, although we admit in this context the need to adapt our approach to realistic electrophysiological models of the cardiac tissue.

This research was supported by the Ministerio de Ciencia y Tecnología (Spain) and FEDER under Projects No. BFM2003-07850, No. BQU2003-05042-C02-01, and No. 2001SGR00045. S.A. was financially supported by a grant from MCyT.
[1] A. T. Winfree, SIAM Rev. 32, 1 (1990).

[2] F. H. Fenton, E. M. Cherry, H. M. Hastings, and S. J. Evans, Chaos 12, 852 (2002).

[3] A. T. Winfree and S. H. Strogatz, Nature (London) 311, 611 (1984).

[4] S. Alonso, F. Sagués, and A. S. Mikhailov, Science 299, 1722 (2003).

[5] S. Alonso, R. Kähler, A. S. Mikhailov, and F. Sagués, Phys. Rev. E 70, 056201 (2004).

[6] R. Kapral and K. Showalter, Chemical Waves and Patterns (Kluwer, Dordrecht, 1995).

[7] J. M. Davidenko, A. V. Pertsov, R. Salomonsz, W. Baxter, and J. Jalife, Nature (London) 355, 349 (1992).

[8] O. Steinbock, F. Siegert, S. C. Muller, and C. J. Weijer, Proc. Natl. Acad. Sci. U.S.A. 90, 7332 (1993).

[9] D. Barkley, M Kness, and L. S. Tuckerman, Phys. Rev. A 42, 2489 (1990).

[10] J. García-Ojalvo and J. M. Sancho, Noise in Spatially Extended Systems (Springer-Verlag, New York, 1999).

[11] S. Kadar, J. C. Wang, and K. Showalter, Nature (London) 391,
770 (1998).

[12] I. Sendiña-Nadal et al., Phys. Rev. Lett. 84, 2734 (2000).

[13] S. Alonso, I. Sendiña-Nadal, V. Pérez-Muñuzuri, J. M. Sancho, and F. Sagués, Phys. Rev. Lett. 87, 078302 (2001).

[14] L. Q. Zhou, X. Jia, and Q. Ouyang, Phys. Rev. Lett. 88, 138301 (2002).

[15] Z. H. Hou and H. W. Xin, Phys. Rev. Lett. 89, 280601 (2002).

[16] S. Alonso, F. Sagués, and J. M. Sancho, Phys. Rev. E 65, 066107 (2002).

[17] J. García-Ojalvo and L. Schimansky-Geier, Europhys. Lett. 47, 298 (1999).

[18] E. Ullner, A. Zaikin, J. García-Ojalvo, and J. Kurths, Phys. Rev. Lett. 91, 180601 (2003).

[19] Y. Zhou and P. Jung, Europhys. Lett. 49, 695 (2000).

[20] V. Pérez-Muñuzuri, F. Sagués, and J. M. Sancho, Phys. Rev. E 62, 94 (2000).

[21] V. N. Biktashev, A. V. Holden, and H. Zhang, Philos. Trans. R. Soc. London, Ser. A 347, 611 (1994).

[22] A. S. Mikhailov, Foundations of Synergetics I: Distributed Active Systems, 2nd ed. (Springer, Berlin, 1996). 